\title{
A Simple Language for Real-Time Cryptographic Protocol Analysis ${ }^{\star}$
}

\author{
Roberto Gorrieri ${ }^{1}$, Enrico Locatelli ${ }^{1}$, and Fabio Martinelli ${ }^{2}$ \\ 1 Dipartimento di Scienze dell’'Informazione, Università di Bologna, Italy. \\ \{gorrieri, locatelli\}@cs.unibo.it \\ 2 Istituto di Informatica e Telematica C.N.R., Pisa, Italy. \\ Fabio.Martinelli@iit.cnr.it
}

\begin{abstract}
A real-time process algebra, enhanced with specific constructs for handling cryptographic primitives, is proposed to model cryptographic protocols in a simple way. We show that some security properties, such as authentication and secrecy, can be re-formulated in this timed setting. Moreover, we show that they can be seen as suitable instances of a general information flow-like scheme, called $t G N D C$, parametric w.r.t. the observational semantics of interest. We show that, when considering timed trace semantics, there exists a most powerful hostile environment (or enemy) that can try to compromise the protocol. Moreover, we hint some compositionality results.
\end{abstract}

\section{Introduction}

In the last years there has been an increasing interest in the formal analysis of cryptographic protocols, as they have become the basic building blocks for many distributed services, such as home banking or electronic commerce. These analyzes have been very successful in many cases, uncovering subtle inaccuracies in many specifications of cryptographic protocols. However, such analyzes are usually restricted to very high abstractions of the real protocols, where concrete information about the timing of events are usually omitted (with the relevant exceptions of $[2,16]$ ).

Our starting point is the work on CryptoSPA [7,9], which is an extension of $S P A$ [4] (a CCS-like process algebra with actions belonging to two different levels of confidentiality), with some new constructs for handling cryptographic primitives. On such a language a general schema for the definition of security properties, called $G N D C$, has been proposed [9]. It is based on the idea of checking the system against all the possible hostile environments. The general schema has the following form:

$$
P \text { satisfies } S_{\triangleleft}^{\alpha} \text { iff } \forall X \in E n v: P \| X \triangleleft \alpha(P)
$$

where the general property $S_{\triangleleft}^{\alpha}$ requires that the system $P$ satisfies (via the behavioral pre-order $\triangleleft$ ) a specification $\alpha(P)$ when composed in parallel with any possible hostile

* Work partially supported by MURST Progetto "Metodi Formali per la Sicurezza" (MEFISTO); IST-FET project "Design Environments for Global ApplicationS (DEGAS)"; Microsoft Research Europe; by CNR project "Tecniche e Strumenti Software per l'analisi della sicurezza delle comunicazioni in applicazioni telematiche di interesse economico e sociale" and by a CSP grant for the project "SeTAPS II". 
environment (or enemy) $X$. The problem of the universal quantification is overcome when it is possible to show that there exists the "most powerful" enemy; hence, one check against the most powerful enemy is as discriminating as an infinity of different checks against all the possible enemies. This lucky case occurs when the behavioral pre-order $\triangleleft$ is a pre-congruence, e.g., for trace semantics.

The main goal of this paper is to show that the real-time information flow theory developed for $t S P A$ (a real-time extension of $S P A$ reported in [8]), can be extended to CryptoSPA, yielding timedCryptoSPA (tCryptoSPA for short). The main results from such an effort are the following:

- A language for describing cryptographic protocols, where information about the concrete timing of events is necessary, e.g., because of the presence of timeouts or time-stamps.

- A general scheme, called $t G N D C$, for describing uniformly the many security properties in a real-time setting; we will present three instances of such a general scheme, namely timed authentication, timed integrity and timed secrecy.

- Some specific results for the security properties based on semantics that are precongruences, such as the existence of a (real-time) most general enemy.

Moreover, we will hint some initial compositionality results, i.e., we will show some conditions under which secure real-time protocols can be safely composed.

The paper is organized as follows: in Section 2 we define the $t$ CryptoSPA syntax, operational and behavioral semantics. In Section 3 we define the general schema $t G N D C$, hence the notion of hostile environment (or enemy) and we present some general results, such as the existence of a real-time most general enemy. In Section 4 we present some security properties, namely $t N D C$, timed authentication, timed integrity and timed secrecy. Section 5 reports some initial results about conditions for safe composition of real-time security protocols. Finally in Section 6 we give some concluding remarks and comparison with related literature.

\section{The Model}

In this section we present the model we will use for the specification of cryptographic protocols and security properties. It is a real-time extension of the Cryptographic Security Process Algebra (CryptoS P A for short) proposed in [9,7], which is in turn an extension of Security Process Algebra (SPA for short) proposed in [4] where processes are explicitly given the possibility of manipulating messages. In CryptoSP $A$ it is possible to express qualitative ordering among events, while quantitative timing aspects cannot be expressed. Thus, we extend CryptoSPA with operators that permit to express the elapsing of time.

\subsection{The Language Syntax}

We call the language Timed Cryptographic Security Process Algebra ( $t$ CryptoSP A for short). Its syntax is based on the following elements:

- A set $C h$ of channels, partitioned into a set $I$ of input channels (ranged over by $c$ ) and a set $O$ of output channels (ranged over by $\bar{c}$, the output corresponding to the input $c)$; 
- A set $\mathcal{M}$ of messages;

- A set $V a r$ of variables, raged over by $x$;

- A set Const of constants, ranged over by $A$.

The set $\mathcal{L}$ of tCryptoSPA terms (or processes) is defined as follows:

$$
\begin{gathered}
P::=\mathbf{0}|c(x) . P| \bar{c} e . P|\tau . P| \text { tick.P }|P+P| P|| P|P \backslash L| \\
A\left(e_{1}, \ldots, e_{n}\right)\left|\left[\left\langle e_{1}, \ldots, e_{r}\right\rangle \vdash_{\text {rule }} x\right] P\right| \iota(P)
\end{gathered}
$$

where $e, e^{\prime}, e_{1}, \ldots, e_{r}$ are messages or variables and $L$ is a set of channels. Both the operators $c(x) . P$ and $\left[\left\langle e_{1} \ldots e_{r}\right\rangle \vdash_{\text {rule }} x\right] P$ bind the variable $x$ in $P$.

Besides the standard (value-passing) CCS operators [15], we have an additional prefix action tick, used to model time elapsing, a delay operator $\iota(P)$, used to make lazy the initial actions of $P$, and the operator $\left[\left\langle m_{1} \ldots m_{r}\right\rangle \vdash_{\text {rule }} x\right] P$ introduced in order to model message handling and cryptography. Informally, process $\left[\left\langle m_{1} \ldots m_{r}\right\rangle \vdash_{\text {rule }} x\right] P$ tries to deduce a piece of information $z$ from the tuple of messages $\left\langle m_{1} \ldots m_{r}\right\rangle$ through one application of rule $\vdash_{\text {rule }}$; if it succeeds, then it behaves like $P[z / x]$, otherwise it is stuck. See the next subsection for a more detailed explanation of derivation rules.

The time model we use is known as the fictitious clock approach of, e.g., [17]. A global clock is supposed to be updated whenever all the processes agree on this, by globally synchronizing on action tick. All the other actions are assumed to take no time. This is reasonable if we choose a time unit such that the actual time of an action is negligible w.r.t. the time unit. Hence, the computation proceeds in lock-steps: between the two global synchronizations on action tick (that represent the elapsing of one time unit), all the processes proceed asynchronously by performing durationless actions.

Let Def : Const $\longrightarrow \mathcal{L}$ be a set of defining equations of the form $A\left(x_{1}, \ldots, x_{n}\right) \stackrel{\text { def }}{=} P$, where $P$ may contain no free variables except $x_{1}, \ldots, x_{n}$, which must be distinct. Constants permit us to define recursive processes, but we have to be a bit careful in using them. A term $P$ is closed w.r.t. Def if all the constants occurring in $P$ are defined in $D e f$ (and, recursively, for their defining terms). A term $P$ is guarded w.r.t. Def if all the constants occurring in $P$ (and, recursively, for their defining terms) occur in a prefix context [15].

The set $A c t=\{c(m) \mid c \in I\} \cup\{\bar{c} m \mid \bar{c} \in O\} \cup\{\tau\} \cup\{t i c k\}$ of actions $(\tau$ is the internal, invisible action, tick is the special action used to model time elapsing), ranged over by $a$ (with abuse of notation); we let $l$ range over $A c t \backslash\{t i c k\}$. We call $\mathcal{P}$ the set of all the $t$ CryptoSPA closed terms (i.e., with no free variables), that are closed and guarded w.r.t. Def. We define $\operatorname{sort}(P)$ to be the set of all the channels syntactically occurring in the term $P$. Moreover, for the sake of readability, we always omit the termination $\mathbf{0}$ at the end of process specifications, e.g., we write $a$ in place of $a .0$.

We give an informal overview of $t C r y p t o S P A$ operators:

- $\mathbf{0}$ is a process that does nothing;

- $c(x) . P$ represents the process that can get an input $m$ on channel $c$ behaving like $P$ where all the occurrences of $x$ are replaced by $m$ (written $P[m / x]$ );

- $\bar{c} m . P$ is the process that can send $m$ on channel $c$, then behaving like $P$;

- $\tau . P$ is the process that executes the invisible action $\tau$ and then behaves like $P$;

- tick.P is a process willing to let one time unit pass and then behaving as $P$; 
- $P_{1}+P_{2}$ (choice) represents the nondeterministic choice between the two processes $P_{1}$ and $P_{2}$; time passes when both $P_{1}$ and $P_{2}$ are able to perform a tick action - and in such a case by performing tick a configuration where both the derivatives of the summands can still be chosen is reached - or when only one of the two can perform tick - and in such a case the other summand is discarded; moreover, $\tau$ prefixed summands have priority over tick prefixed summands.

- $P_{1} \| P_{2}$ (parallel) is the parallel composition of processes that can proceed in an asynchronous way but they must synchronize on complementary actions to make a communication, represented by a $\tau$. Both components must agree on performing a tick action, and this can be done even if a communication is possible;

- $P \backslash L$ is the process that cannot send and receive messages on channels in $L$, for all the other channels it behaves exactly like $P$;

- $A\left(m_{1}, \ldots, m_{n}\right)$ behaves like the respective defining term $P$ where all the variables $x_{1}, \ldots, x_{n}$ are replaced by the messages $m_{1}, \ldots, m_{n}$;

- $\left[\left\langle m_{1}, \ldots, m_{r}\right\rangle \vdash_{\text {rule }} x\right] P$ is the process used to model message handling and cryptography. The process $\left[\left\langle m_{1}, \ldots, m_{r}\right\rangle \vdash_{\text {rule }} x\right] P$ tries to deduce an information $z$ from the tuple of messages $\left\langle m_{1}, \ldots, m_{r}\right\rangle$ through the application of rule $\vdash_{\text {rule }}$; if it succeeds then it behaves like $P[z / x]$, otherwise it is stuck. The set of rules that can be applied is defined through an inference system (e.g., see Figure 1);

- $\iota(P)$ (idling) allows process $P$ to wait indefinitely. At every instant of time, if process $P$ performs an action $l$, then the whole system proceeds in this state, while dropping the idling operator.

\subsection{The Operational Semantics of tCryptoSPA}

In order to model message handling and cryptography we use a set of inference rules. Note that $t$ CryptoSPA syntax, its semantics and the results obtained are completely parametric with respect to the inference system used. We present in Figure 1 the same inference system of [9]. This inference system can combine two messages obtaining a pair (rule $\vdash_{\text {pair }}$ ); it can extract one message from a pair (rules $\vdash_{f s t}$ and $\vdash_{s n d}$ ); it can encrypt a message $m$ with a key $k$ obtaining $\{m\}_{k}$ and finally decrypt a message of the form $\{m\}_{k}$ only if it has the same key $k$ (rules $\vdash_{e n c}$ and $\vdash_{d e c}$ ). In this framework, cryptography is completely reliable, i.e., that a crypted message can be deciphered only by knowing the suitable decryption key.

In a similar way, the inference system can contain rules for handling the basic arithmetic operations and boolean relations among numbers, so that the value-passing CCS if-then-else construct can be obtained via the $\vdash_{\text {rule }}$ operator.

Example 1. We do not explictly define equality check among messages in the syntax. However, this can be implemented through the usage of the inference construct. E.g., consider rule equal $\stackrel{\text { def }}{=} \frac{x x}{\operatorname{Equal}(x, x)}$. Then $\left[m=m^{\prime}\right] A$ (with the expected semantics) may be equivalently expressed as $\left[\begin{array}{lll}m & m^{\prime} \vdash_{\text {equal }} & y\end{array}\right] A$ where $y$ does not occur in $A$. Similarly, we can define inequalities, e.g., $\leq$, among natural numbers.

We consider a function $\mathcal{D}$, from finite sets of messages to sets of messages, such that $\mathcal{D}(\phi)$ is the set of messages that can be deduced from $\phi$ by using the inference rules. We assume that $\mathcal{D}$ is decidable. 


$$
\begin{gathered}
\frac{m m^{\prime}}{\left(m, m^{\prime}\right)}\left(\vdash_{\text {pair }}\right) \frac{\left(m, m^{\prime}\right)}{m}\left(\vdash_{f s t}\right) \frac{\left(m, m^{\prime}\right)}{m^{\prime}}\left(\vdash_{s n d}\right) \\
\frac{m k}{\{m\}_{k}}\left(\vdash_{e n c}\right) \quad \frac{\{m\}_{k} k}{m}\left(\vdash_{\text {dec }}\right)
\end{gathered}
$$

Fig. 1. An example inference system for shared key cryptography.

The operational semantics of a $t C r y p t o S P A$ term is described by means of the labelled transition system (lts, for short) $\left\langle\mathcal{P}, A c t,\{\stackrel{a}{\longrightarrow}\}_{a \in A c t}\right\rangle$, where $\{\stackrel{a}{\longrightarrow}\}_{a \in A c t}$ is the least relation between $t C r y p t o S P A$ processes induced by the axioms and inference rules of Figure 2. Such a relation is well-defined even if negative premises occur in a rule for the idling operator and in one rule for + , because the relation is strictly stratifiable [12].

Note that $t$ CryptoSPA is tick-deterministic i.e., the time elapsing never moves a process to two different states. The proof of the following proposition can be easily given by inspecting the operational rules. In particular, the first two rules of the idling operator and the rules for nondeterministic choice are the key rules enforcing time determinacy.

Proposition 1. For every tCryptoSPA process $P$ we have:

If $P \stackrel{\text { tick }}{\longrightarrow} P^{\prime}$ and $P \stackrel{\text { tick }}{\longrightarrow} P^{\prime \prime}$ then $P^{\prime}=P^{\prime \prime}$.

Example 2. In $t$ CryptoSPA there are processes, such as $\mathbf{0}$, that do not allow time to proceed; hence, as rule $\|_{3}$ for parallel composition forces a global synchronisation on tick actions, the effect of composing a process $P$ with $\mathbf{0}$ is to prevent $P$ from letting time pass. In other words, $\mathbf{0}$ acts as a time annihilator for its parallel context. On the contrary, $\iota(\mathbf{0})$ is process that, even if functionally terminated, let time to proceed indefinitely. Hence, $\iota(\mathbf{0})$ acts as a neutral element for parallel composition.

Example 3. Consider a process $P=\iota(a) \| \iota(\bar{a})$ that can perform any sequence (possibly empty) of tick actions followed by a $\tau$. It is worth-observing that, contrary to $t S P A$ [8], we do not assume maximal communication progress, i.e., $\tau$ 's do not have priority over tick actions or, equivalently, a process cannot idle if it can perform a $\tau$. Hence in $t S P A$ process $P$ can perform only the sequence $\tau$.

Example 4. We can easily model timeout constructs in tCryptoSPA.

Assume $n_{1} \leq n_{2}$ and define a process

$$
\text { Time_out }\left(n_{1}, n_{2}, A, B\right)=t i c k^{n_{1}} \cdot \iota(A)+t i c k^{n_{2}} \cdot \tau . B
$$

By looking at the rules for choice and idling, we see that Time_out $\left(n_{1}, n_{2}, A, B\right)$ first performs a sequence of $n_{1}$ tick actions; then, the system may perform other $n_{2}-n_{1} t i c k$ actions, unless $A$ resolves the choice by performing an action; instead if $A$ does nothing, after $n_{2}$ units of time, through the execution of action $\tau$, the process is forced to act as $B$. Note that rule $+_{3}$ is responsible for preventing the selection of process $A$ at timeout expiration. This semantics for the + operator is different from the one in $t S P A$ (a tick action can be performed only if both summands can do so) and is motivated by the need of a more flexible way of programming the choice between different components. 


$$
\begin{aligned}
& (\text { input }) \frac{m \in \mathcal{M}}{c(x) \cdot P \stackrel{c(m)}{\longrightarrow} P[m / x]} \quad \text { (output) } \frac{}{\bar{c} m \cdot P \stackrel{\bar{c} m}{\longrightarrow} P} \\
& (\text { internal }) \underset{\tau . P \stackrel{\tau}{\longrightarrow} P}{\longrightarrow} \quad(\text { tick })-\frac{\text { tick.P } \stackrel{\text { tick }}{\longrightarrow} P}{\text { ․ }}
\end{aligned}
$$

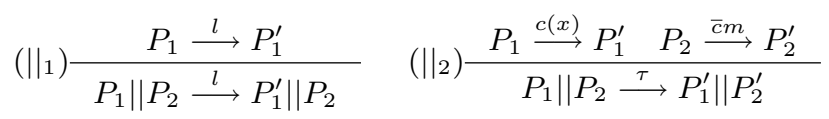

$$
\begin{aligned}
& \left(\|_{3}\right) \frac{P_{1} \stackrel{\text { tick }}{\longrightarrow} P_{1}^{\prime} \quad P_{2} \stackrel{\text { tick }}{\longrightarrow} P_{2}^{\prime}}{P_{1}\left\|P_{2} \stackrel{\text { tick }}{\longrightarrow} P_{1}^{\prime}\right\| P_{2}^{\prime}} \quad(\backslash L) \frac{P \stackrel{c(m)}{\longrightarrow} P^{\prime} \quad c \notin L}{P \backslash L \stackrel{c(m)}{\longrightarrow} P^{\prime} \backslash L} \\
& (+1) \frac{P_{1} \stackrel{l}{\longrightarrow} P_{1}^{\prime}}{P_{1}+P_{2} \stackrel{l}{\longrightarrow} P_{1}^{\prime}} \quad\left(++_{2}\right) \frac{P_{1} \stackrel{\text { tick }}{\longrightarrow} P_{1}^{\prime} \quad P_{2} \stackrel{\text { tick }}{\longrightarrow} P_{2}^{\prime}}{P_{1}+P_{2} \stackrel{\text { tick }}{\longrightarrow} P_{1}^{\prime}+P_{2}^{\prime}} \\
& (+3) \frac{P_{1} \stackrel{\text { tick }}{\longrightarrow} P_{1}^{\prime} \quad P_{2} \stackrel{\text { tjck }}{\longrightarrow} P_{2} \stackrel{\tau}{\longrightarrow}}{P_{1}+P_{2} \stackrel{\text { tick }}{\longrightarrow} P_{1}^{\prime}} \\
& (D e f) \frac{P\left[m_{1} / x_{1}, \ldots, m_{n} / x_{n}\right] \stackrel{a}{\longrightarrow} P^{\prime} A\left(x_{1}, \ldots, x_{n}\right) \stackrel{\text { def }}{=} P}{A\left(m_{1}, \ldots, m_{n}\right) \stackrel{a}{\longrightarrow} P^{\prime}} \\
& (\mathcal{D}) \frac{\left\langle m_{1}, \ldots, m_{r}\right\rangle \vdash_{\text {rule }} m \quad P[m / x] \stackrel{a}{\longrightarrow} P^{\prime}}{\left[\left\langle m_{1}, \ldots, m_{r}\right\rangle \vdash_{\text {rule }} x\right] P \stackrel{a}{\longrightarrow} P^{\prime}} \\
& \left(\mathcal{I}_{1}\right) \frac{P \stackrel{\text { tjck }}{\longrightarrow}}{\iota(P) \stackrel{\text { tick }}{\longrightarrow} \iota(P)}\left(\mathcal{I}_{2}\right) \frac{P \stackrel{\text { tick }}{\longrightarrow} P^{\prime}}{\iota(P) \stackrel{\text { tick }}{\longrightarrow} \iota\left(P^{\prime}\right)} \quad\left(\mathcal{I}_{3}\right) \frac{P \stackrel{l}{\longrightarrow} P^{\prime}}{\iota(P) \stackrel{l}{\longrightarrow} P^{\prime}}
\end{aligned}
$$

Fig. 2. Structured Operational Semantics for tCryptoSPA (symmetric rules for $+_{1},+_{3},\|\|_{1},\|\|_{2}$ and $\backslash L$ are omitted)

\section{A General Schema for the Definition of Timed Security Properties}

In this section we propose a general schema for the definition of timed security properties. We call it Timed Generalized $N D C$ ( $t G N D C$ for short), since it is a real-time generalization of Generalized $N D C$ ( $G N D C$ for short) [9], which is in turn a generalization of Non Deducibility on Compositions ( $N D C$ for short) [4]. The main idea is the following: a system $P$ is $t G N D C_{\triangleleft}^{\alpha}$ iff for every hostile environment (or enemy) $X$ the composition of the system $P$ with $X$ satisfies the timed specification $\alpha(P)$. Essentially $t G N D C_{\triangleleft}^{\alpha}$ guarantees that the timed property $\alpha$ is satisfied, with respect to the $\triangleleft$ timed behavioral relation, even when the system is composed with any possible enemy.

This section is organized as follows. We first define timed trace semantics as the behavioral semantics of interest. Then, we discuss the issue of hostile environments, showing that it is necessary to restrict their initial knowledge. Finally, we present the $t G N D C$ schema and some general results on it, some of which are independent of the chosen behavioral notion. 


\subsection{Behavioural Semantics}

Here we define the semantic pre-order and equivalence we will use to formalize security properties, timed trace pre-order and equivalence, the timed version of the classic untimed semantics.

The expression $P \stackrel{a}{\Rightarrow} P^{\prime}$ is a shorthand for $P(\stackrel{\tau}{\longrightarrow})^{*} P_{1} \stackrel{a}{\longrightarrow} P_{2}(\stackrel{\tau}{\longrightarrow})^{*} P^{\prime}$ where $(\stackrel{\tau}{\longrightarrow})^{*}$ denotes a (possibly empty) sequence of transitions labeled $\tau$. Let $\gamma=a_{1}, \ldots, a_{n} \in$ $(A c t \backslash\{\tau\})^{*}$ be a sequence of actions; then $P \stackrel{\gamma}{\Rightarrow} P^{\prime}$ iff there exist $P_{1}, \ldots, P_{n-1} \in \mathcal{P}$ such that $P \stackrel{a_{1}}{\Rightarrow} P_{1} \stackrel{a_{2}}{\Rightarrow}, \ldots, P_{n-1} \stackrel{a_{n}}{\Rightarrow} P^{\prime}$.

Definition 1. For any $P \in \mathcal{P}$ the set $T(P)$ of timed traces associated with $P$ is defined as follows $T(P)=\left\{\gamma \in(\text { Act } \backslash\{\tau\})^{*} \mid \exists P^{\prime} . P \stackrel{\gamma}{\Rightarrow} P^{\prime}\right\}$. The timed trace pre-order, denoted by $\leq_{\text {ttrace }}$, is defined as follows: $P \leq_{\text {ttrace }} Q$ iff $T(P) \subseteq T(Q) . P$ and $Q$ are timed trace equivalent, denoted by $P={ }_{\text {ttrace }} Q$, if $T(P)=T(Q)$.

As an example, it is easy to see that $T\left(P\left(K_{a b}\right)\right)=\{\epsilon$, tick, tick tick, tick tick tick $\}$, where $\epsilon$ denotes the empty sequence.

\subsection{Hostile Environments}

Here we characterize the notion of admissible hostile environments similarly to what done in [9] for the untimed setting. Such a characterization is necessary to analyze protocols where some information is assumed to be secret, as in cryptographic protocols. A hostile environment, or enemy, is a process which tries to attack a protocol by stealing and faking information which is transmitted on public channels, say $C$. Such an agent is modeled as a generic process $X$ which can communicate only through channels in $C$, imposing some constraints on the initial data that are known by the enemy and requiring that such a protocol is weakly time alive, i.e., the agent may always perform tick eventually. Otherwise $X$ could prevent time from elapsing when composed in parallel with some system, since in a compound system time can pass iff all components let it pass. So the enemy could block the time flow and we want to avoid this unrealistic case. Let $\operatorname{Der}(P)$ be the set of all derivatives of $P$, i.e., all the $P^{\prime}$ s reachable from $P$ through a sequence of actions in Act.

Definition 2. A process $P$ is directly weakly time alive iff $P \stackrel{\text { tick }}{\Longrightarrow} P^{\prime} . P$ is weakly time alive iff for all $P^{\prime} \in \operatorname{Der}(P)$, we have $P^{\prime}$ is directly weakly time alive.

Now, let $I D(P)$ be the set of messages that appear in $P$ (see [5] for a formal definition) and $\phi \subseteq \mathcal{M}$ be the initial knowledge we would like to give to the enemies, i.e., the public information such as the names of the entities and the public keys, plus some possible private data of the enemies (e.g., their private key or nonces). For some enemy $X$, we want that all the messages in $I D(X)$ are deducible from $\phi$. We thus define the set $t \mathcal{E}_{C}^{\phi_{I}}$ of timed hostile processes as:

$$
t \mathcal{E}_{C}^{\phi}=\{X \in \mathcal{P} \mid \operatorname{sort}(X) \subseteq C \text { and } I D(X) \subseteq \mathcal{D}(\phi) \text { and } X \text { is weakly time alive }\}
$$

\subsection{The $t G N D C$ Schema}

In this section we formally define the $t G N D C_{\triangleleft}^{\alpha}$ family of properties. We will use $A \|_{C} B$ as a shortcut for $(A \| B) \backslash C$. The proposed family of security properties is as follows. 
Definition 3. $P$ is $t G N D C_{\triangleleft}^{\alpha}$ iff $\forall X \in t \mathcal{E}_{C}^{\phi_{I}}:\left(P \|_{C} X\right) \triangleleft \alpha(P)$ where $\triangleleft \in \mathcal{P} \times \mathcal{P}$ is a pre-order, $C$ is a set of channels and $\alpha: \mathcal{P} \mapsto \mathcal{P}$ is a function between processes defining the property specification for $P$ as the process $\alpha(P)$.

We propose a sufficient criterion for a static characterization (i.e., not involving the universal quantification $\forall$ ) of $t G N D C_{\triangleleft}^{\alpha}$ properties. We will say that $\triangleleft$ is a pre-congruence w.r.t. $\|_{C}$ if it is a pre-order and for every $P, Q, Q^{\prime} \in \mathcal{P}$ if $Q \triangleleft Q^{\prime}$ then $P\left\|_{C} Q \triangleleft P\right\|_{C} Q^{\prime}$. Thus it is easy to prove the following:

Proposition 2. If $\triangleleft$ is a pre-congruence w.r.t. $\|_{C}$ and if there exist a process Top $\in$ t政 such that for every process $X \in t_{C}^{\phi_{I}}$ we have $X \triangleleft T o p$, then $\forall \alpha$ :

$$
P \in t G N D C_{\triangleleft}^{\alpha} \quad \text { iff } \quad\left(P \|_{C} T o p\right) \triangleleft \alpha(P)
$$

In particular if the hypotheses of the proposition above hold it is sufficient to check that $\alpha(P)$ is satisfied when $P$ is composed with the most general hostile environment Top. This permits to make only one single check, in order to prove that a property holds whatever attacker we choose. We also have the following corollary for the congruence induced by $\triangleleft$ :

Corollary 1. Let $\triangleleft$ be a pre-congruence w.r.t. $\|_{C}$ and let $\equiv=\triangleleft \cap \triangleleft^{-1}$. If there exist two processes Bot, Top $\in t \mathcal{E}_{C}^{\phi_{I}}$ such that for every process $X \in t \mathcal{E}_{C}^{\phi_{I}}$ we have Bot $\triangleleft X \triangleleft T$ Top then

$$
P \in t G N D C_{\equiv}^{\alpha} \quad \text { iff }\left(P \|_{C} B o t\right) \equiv\left(P \|_{C} T o p\right) \equiv \alpha(P)
$$

Given these very general results, we show that they are instanciable in the model we presented so far. Indeed, this is the case, at least for the trace pre-order $\leq_{\text {trace }}$, which is a pre-congruence.

Proposition 3. Timed trace pre-order is a pre-congruence w.r.t. $\|_{C}$.

Note that in the $t S P A$ model, timed trace pre-order is not a pre-congruence, since the semantic rules enforce the so called maximal communication progress, i.e., when a communication is possible it must start immediately, and it is not possible to perform a tick [8].

Now we single out the minimal element Bot and the maximum element Top in $t \mathcal{E}_{C}^{\phi_{I}}$ w.r.t. $\leq_{\text {trace }}$. As for Bot it is clear that the minimum set of traces is generated by the weakly time alive process that does nothing, that is generated by process $\iota(\mathbf{0})$. As a matter of fact, $(P \| \iota(\mathbf{0}))={ }_{\text {ttrace }} P$ for timed trace equivalence and most other equivalences. We thus define the Top element using a family of processes Top triace each representing an instance of the enemy with knowledge $\phi$ :

$$
\text { Top ttrace }_{\text {tro }}^{C, \phi}=\sum_{c \in C} \iota\left(c(x) \cdot \text { Top ttrace }_{\text {tra }}^{C, \phi \cup\{x\}}\right)+\sum_{c \in C, m \in \mathcal{D}(\phi) \cap \mathcal{M}} \iota(\bar{c} \text { m.Top ttrace })
$$

The initial element of the family is $\operatorname{Top}_{\text {ttrace }}^{C, \phi_{I}}$ as $\phi_{I}$ is the initial knowledge of the enemy. This may accept any input message to be bound to the variable $x$ which is then added to the knowledge set that becomes $\phi_{I} \cup\{x\}$, and may output only messages that can pass on the channel $c$ and that are deducible from the current knowledge set $\phi$ via the deduction function $\mathcal{D}$. Furthermore it can let time pass. Note that $\tau$ summands are not considered, as inessential for trace pre-order. As done in [9] we prove the following:

Proposition 4. If $X \in t \mathcal{E}_{C}^{\phi}$ then $X \leq_{\text {ttrace }}$ Top ttrace. 


\section{Some Timed Security Properties}

In this section we show how to redefine four timed security properties as suitable instances of the $t G N D C_{\triangleleft}^{\alpha}$ schema, by suitably defining function $\alpha$. As for the behavioral semantics $\triangleleft$, we will always consider the timed trace semantics. The four properties we consider are:

- The timed version of Non Deducibility on Compositions [4], which has been proposed to study information flow security; we will show that $t N D C$ is the strongest property in the $t G N D C_{\triangleleft}^{\alpha}$ family, under some mild assumptions.

- A timed notion of authentication, called timed agreement (see also [14]), according to which agreement must be reached within a certain deadline, otherwise authentication does not hold.

- A timed notion of secrecy, we call timed secrecy, according to which a message is secret only within a time interval and after the deadline it can become a public piece of information.

- A timed notion of integrity, called timed integrity, which simply requires a correct delivery of messages within a certain amount of time.

\subsection{Timed Non Deducibility on Compositions}

We start with $t N D C$ since $t G N D C_{\triangleleft}^{\alpha}$ is a generalization of such a property. Its underlying idea is that the system behavior must be invariant w.r.t. the composition with every hostile environment. Indeed, there is no possibility of establishing a communication (i.e. sending information). In the CryptoSPA untimed setting the $N D C^{1}$ idea can thus be defined as follows:

Definition 4. $P \in N D C$ if and only if $\forall X \in \mathcal{E}_{C}^{\phi_{I}}$, we have $\left(P \|_{C} X\right)=_{\text {trace }} P \backslash C$.

where ${ }_{\text {trace }}$ is trace pre-order and the only difference with the definition given in SPA is that the knowledge of process $X$ is bounded by $\phi_{I}$. Now we present timed $N D C$ ( $t N D C$, for short) ([8]) which is the natural extension of $N D C$ to a timed setting.

Definition 5. $P \in t N D C$ if and only if $\forall X \in t \mathcal{E}_{C}^{\phi_{I}}$ we have $\left(P \|_{C} X\right)=_{\text {trace }} P \backslash C$. where the difference is that we use the timed hostile environment and timed trace preorder. Note that $t N D C$ corresponds to $t G N D C{ }_{=t \text { trace }}^{P \backslash C}$. It is also possible to apply Corollary 1 obtaining the following static characterization.

Proposition 5. $P \in t N D C$ if and only if $\left(P \|_{C}\right.$ Top trace $\left.{ }_{\text {trac }}^{C}\right)={ }_{\text {trace }} P \backslash C$.

Now we suggest that $t N D C$ is the most restrictive $\alpha(P)$ hence inducing the strongest property for timed trace semantics. The most restrictive $\alpha(P)$ should return an encapsulation of protocol $P$, i.e., a version of $P$ which is completely isolated from the environment, corresponding to the execution of $P$ in a perfectly secure network where only the honest parties are present. In our process algebra setting, this corresponds to the restriction of all public channels in $C$ along which protocol messages are sent.

Note that for every process $P$ we have $(P \| \iota(\mathbf{0})) \backslash C={ }_{\text {ttrace }} P \backslash C$. This means that $P$ restricted on $C$ is equivalent to the protocol in composition with the enemy that

\footnotetext{
${ }^{1}$ As for $t G N D C_{\triangleleft}^{\alpha}$, also $N D C$ and $t N D C$ are implicitly parametric w.r.t. the set $C$ of public channels and the set $\phi_{I}$ of initial knowledge. We usually omit these parameters when clear from the context.
} 
does nothing. Note also that, by definition, $\iota(\mathbf{0}) \in t \mathcal{E}_{C}^{\phi}$ for every $\phi$. So it is very natural to consider $\alpha$ functions and processes $P$ such that $P \backslash C \leq_{\text {trace }} \alpha(P)$. This simply means that the protocol $P$ is correct (as it satisfies its specification $\alpha(P)$ ) at least when it is not under attack. This condition can be somehow seen as a reasonable criterion for any good protocol: it must be correct at least when it is not under attack! Under this mild assumption, it is clear that $P \in t N D C$ implies $P \in t G N D C_{\leq_{\text {ttrace }}}^{\alpha}$.

Another way to avoid universal quantification over all the admissible enemies is to show the equivalence between $t N D C$ and Timed Strong Nondeterministic NonInterference ( $t S N N I$, for short); such equivalence result holds in the untimed case [4], but that does not hold for $t S P A[8]$ because of the maximal communication assumption of that language.

A CryptoSPA process is $S N N I_{C}^{\phi}$ if $P \backslash C$, where all actions in $C$ are forbidden, behaves like the system $P$ where all the actions in $C$ are hidden (i.e., transformed into internal $\tau$ actions). To express this second system we need to introduce first the hiding operator $P /{ }^{\phi} C$ :

$$
\begin{aligned}
& \frac{P \stackrel{a}{\longrightarrow} P^{\prime}}{P / \phi C \stackrel{a}{\longrightarrow} P^{\prime} / \phi C}(a \notin C \cup \bar{C}) \frac{P \stackrel{\bar{c} m}{\longrightarrow} P^{\prime} \quad c \in C \cup \bar{C}}{P /{ }^{\phi} C \stackrel{\tau}{\longrightarrow} P^{\prime} / \phi \cup\{m\}} C \\
& \frac{P \stackrel{c(m)}{\longrightarrow} P^{\prime} \quad c \in C \cup \bar{C} \quad m \in \mathcal{D}(\phi)}{P /{ }^{\phi} C \stackrel{\tau}{\longrightarrow} P^{\prime} /{ }^{\phi} C}
\end{aligned}
$$

Now we are ready to define the property timed $S N N I_{C}^{\phi}$ as follows.

Definition 6. A process is $t S N N I_{C}^{\phi}$ if $P \backslash C={ }_{\text {trace }} P / \phi C$.

It is rather intuitive that $P /{ }^{\phi} C$ can be seen as $P \|_{C} T o p$, where Top is the top element of the trace pre-order for CryptoSPA. Hence, such a static characterization can be seen as a corollary of the existence of a top element in the trace pre-order (together with the fact that trace pre-order is a pre-congruence).

Proposition 6. For every $P \in \mathcal{P}$ we have that $\left(P \|_{C}\right.$ Top ttrace ${ }_{\text {trace }}^{C, \phi} P{ }^{\phi} C$.

Proposition 7. $P \in t N D C_{C}^{\phi}$ iff $P \in t S N N I_{C}^{\phi}$.

\subsection{Timed Agreement}

We now present the Timed Agreement Property [14]:

"A protocol guarantees Timed Agreement between a responder $B$ and an initiator $A$ on a set of data items $d s$ if, whenever $B$ (acting as responder) completes a run of the protocol, apparently with initiator $A$, then $A$ has previously been running the protocol, apparently with $B$, in the last $n$ ticks (where $n$ is a prefixed timeout value) and the two agents agreed on the data values corresponding to all the variables in $d s$, and each such a run of $B$ corresponds to a unique run of A."

As done in [9] for the non real-time version of Agreement, what we do is to have for each party an action representing the running of a protocol and another one representing the completion of it. We consider an action commit_res $(B, A, d)$ representing a correct 
termination of $B$ as a responder, convinced to communicate with $A$ that agrees on data $d$. On the other hand we have an action running_ini $(A, B, d)$ that represents the fact that $A$ is running the protocol as initiator, apparently with $B$, with data $d$. If we specify these two actions in the protocol, the Timed Agreement property requires that when $B$ executes commit_res $(B, A, d)$ then $A$ has previously executed running_ini $(A, B, d)$ and at most $n$ tick actions, where $n$ is the prefixed timeout value, occurred between these actions. We assume that the actions representing the running and the commit are correctly specified in the protocol. We can see them as output actions over two particular channels running_ini and commit_res. We assume that $d$ can assume values in a set $D$. Let $\operatorname{NotObs}(P)=\operatorname{sort}(P) \backslash(C \cup\{$ running_ini, commit_res $\})$ be the set of channels in $P$ that are not public and are different from running_ini and commit_res, i.e., that will not be observed. Function $\alpha_{t \text { Agree }}^{t(n)}$ can be thus defined as follows:

$$
\begin{aligned}
& P^{\prime}(x, y)=\sum_{d \in D, i \in 0 . . n} \iota\left(\overline{\text { running_ini }}(x, y, d) . t i c k_{1} . . t i c k_{i} . \overline{\text { commit_res }_{1}}(y, x, d) . \iota(\mathbf{0})\right) \\
& P^{\prime \prime}=\sum_{c \in N o t O b s(P)} \iota\left(c(x) \cdot P^{\prime \prime}\right)+\sum_{c \in N o t O b s(P), m \in \mathcal{M}} \iota\left(\bar{c} m . P^{\prime \prime}\right) \\
& \alpha_{\text {tAgree }}^{t(n)}(P)=P^{\prime \prime} \| P^{\prime}(A, B)
\end{aligned}
$$

Note that $P^{\prime \prime}$ is essentially the process that executes every possible action over channels in $\operatorname{sort}(P)$ which are not in $C$ and are different from running_ini and commit_res, or let time pass. Given $P, \alpha_{\text {tAgree }}^{t(n)}(P)$ represents the most general system which satisfies the Timed Agreement property and has the same sort of $P$. In fact in $\alpha_{t \text { Agree }}^{t(n)}(P)$ action $\overline{\text { running_ini }}(x, y, d)$ always precedes $\overline{c o m m i t \_r e s}(y, x, d)$ for every datum $d$, and every combination of the other actions of $P$ can be executed. Finally the number of tick actions is at most $n$. In order to analyze more than one session, it suffices to consider an extended $\alpha$ which has several processes $P^{\prime}$ in parallel.

We want that even in the presence of a hostile process, $P$ does not execute traces that are not in $\alpha_{\text {tAgree }}^{t(n)}(P)$. So we can give the following definition:

Definition 7. $P$ satisfies Timed Agreement iff $P$ is $t G N D C_{\leq_{\text {ttrace }}}^{\alpha_{\text {tagree }}^{(n)}(P)}$, i.e.,

$$
\forall X \in t \mathcal{E}_{C}^{\phi_{I}}:\left(P \|_{C} X\right) \leq_{\text {ttrace }} \alpha_{\text {tAgree }}^{t(n)}(P)
$$

\subsection{Timed Secrecy}

We now present the Timed Secrecy Property:

"A protocol guarantees to an initiator $A$ the property of Timed Secrecy on a set of data items $d s$ within a time $n$ if, whenever a data item in $d s$ becomes public, at least $n$ ticks passed since $A$ started the protocol"

As done for Timed Agreement, what we do is to have an action representing the running of a protocol and another one representing that a secret is revealed. We consider an action running_ini $(A, d)$ that represents the fact that $A$ is running the protocol as initiator, with data $d$. On the other hand we have an action $\overline{p u b l i c}(d)$ representing that data item $d$ is made public. If we specify these two actions in the protocol, the Timed Secrecy property requires that when someone executes $\overline{p u b l i c}(d)$ then $A$ has executed running_ini $(A, d)$ and at least $n$ tick actions, where $n$ is the prefixed timeout value, 
occurred between them. We assume that the actions representing the running and the publication are correctly specified in the protocol. We can see the first as an output action over a particular channel running_ini. The second action, following the approach of [6] is performed by a particular process called Key Expired Notifier (KEN, for short) that reads from a public channel $c$ not used in the protocol and performs the output of what it has read on the channel $\overline{\text { public }}$, i.e. $K E N=c(x) \cdot \overline{p u b l i c}(x)$.

Let $N o t O b s(P)=\operatorname{sort}(P) \backslash(C \cup\{c$, running_ini, public $\})$ be the set of channels in $P$ that are not public and are different from running_ini and public, i.e., that will not be observed. We assume that $d$ can take values in a set of secret values $D$. Function $\alpha_{t S e c}^{t(n)}$ can be thus defined as follows:

$$
\begin{aligned}
& P^{\prime}(x)=\sum_{d \in D} \iota\left(\overline{\text { running_ini }}(x, d) \cdot \text { tick }_{1} \ldots \text { tick }_{n} \cdot(\iota(\overline{\text { public }}(d) . \iota(\mathbf{0}))+\iota(\tau . \iota(\mathbf{0})))\right) \\
& P^{\prime \prime}=\sum_{c \in N o t O b s(P)} \iota\left(c(x) \cdot P^{\prime \prime}\right)+\sum_{c \in N o t O b s(P), m \in \mathcal{M}} \iota\left(\bar{c} m . P^{\prime \prime}\right) \\
& \alpha_{t S e c}^{t(n)}(P)=P^{\prime \prime} \| P^{\prime}(A)
\end{aligned}
$$

Given $P, \alpha_{t S e c}^{t(n)}(P)$ represents the most general system which satisfies the Timed Secrecy property and has the same sort of $P$. In fact in $\alpha_{t S e c}^{t(n)}(P)$ action $\overline{p u b l i c}(d)$ is always executed at least $n$ ticks after $\overline{\text { running_ini }}(x, d)$ for every datum $d$, and every combination of the other actions of $P$ can be executed. In order to analyze more than one session, it suffices to consider an extended $\alpha$ which has several processes $P^{\prime}$ in parallel.

We want that, even in the presence of a hostile process, $P$ does not execute traces that are not in $\alpha_{t S e c}^{t(n)}(P)$. So we can give the following definition:

Definition 8. P satisfies Timed Secrecy iff $P$ is $t G N D C_{\leq t \text { trace }}^{\alpha_{t S e c}^{t(n)}(P)}$, i.e.,

$$
\forall X \in t \mathcal{E}_{C}^{\phi_{I}}:\left(P \|_{C} X\right) \leq_{\text {ttrace }} \alpha_{t S e c}^{t(n)}(P)
$$

\subsection{Timed Integrity}

We now present the Timed Integrity Property:

"A protocol guarantees to the user $B$ the property of Timed Integrity on a set of data items $d s$ within a time $n$ if $B$ only accepts data items in $d s$ and this may only happen in at most $n$ ticks since the beginning of the protocol"

For instance, imagine that you would like to receive your favorite newspaper each day before noon. This may be expressed as an integrity property rather than an authenticity one, since you are not actually interested in the sender but simply on the data (the newspaper). Consider a channel out used for expressing the reception of a message and let $N o t O b s(P)=\operatorname{sort}(P) \backslash(C \cup\{$ out $\})$ be the set of channels in $P$ that are not public and $d$ ranging over a set of data $D$. Then, Timed Integrity may be formally specified as follows:

$$
\begin{aligned}
& P^{\prime}(y, n)=\|_{d \in D} \text { tick }_{1} \ldots . t i c k_{n} \cdot \tau \cdot \iota(\mathbf{0})+\iota(\overline{\text { out }}(y, d) \cdot \iota(\mathbf{0})) \\
& P^{\prime \prime}=\sum_{c \in N o t O b s(P)} \iota\left(c(x) \cdot P^{\prime \prime}\right)+\sum_{c \in N o t O b s}(P), m \in \mathcal{M} \iota\left(\bar{c} m . P^{\prime \prime}\right) \\
& \alpha_{t I n t}^{t(n)}(P)=P^{\prime \prime} \| P^{\prime}(B, n)
\end{aligned}
$$




\section{Compositionality Results}

In this section we illustrate some compositional proof rules for establishing that a system enjoys a $t G N D C$ property, in particular $t S N N I_{C}^{\phi}$. However, remember that, as it is equivalent to $t N D C$, this property implies all the other ones based on trace semantics, that are the most frequently used in security analysis.

Within the $S P A$ theory, $S N N I$ is compositional, i.e. if $P, Q \in S N N I$ then $(P \| Q) \in S N N I$. Unfortunately, the same does not hold when considering enemies with limited knowledge, as for $t S N N I_{C}^{\phi}$. For instance, consider the processes:

$$
P=\overline{c_{1}} m_{1} \cdot c_{2}(x)\left[x=m_{2}\right] \cdot \overline{c_{3}} m_{2} \quad Q=\overline{c_{1}} m_{2} \cdot c_{2}(x)\left[x=m_{1}\right] \cdot \overline{c_{3}} m_{1}
$$

Now, assuming $C=\left\{c_{1}, c_{2}\right\}$ and $\phi=\emptyset$, we have that $P, Q \in t S N N I_{C}^{\phi}$. However, $P \| Q \notin t S N N I^{\phi}$. As a matter of fact, $(P \| Q) \backslash C$ is equivalent to $\mathbf{0}$, while $(P \| Q){ }^{\phi} C$ may perform both $\overline{c_{3}} m_{1}$ and $\overline{c_{3}} m_{2}$.

However, if we strengthen the assumptions we can get a compositional rule for establishing that a process belongs to $t S N N I_{C}^{\phi}$. The stability assumption we make is that the process cannot increment its knowledge.

Definition 9. We say that a process $P$ is stable w.r.t. $\phi$, whenever if $P /{ }^{\phi} C \Longrightarrow P^{\prime} / \phi^{\prime} C$ then $\mathcal{D}(\phi)=\mathcal{D}\left(\phi^{\prime}\right)$.

Thus, the following proposition holds.

Proposition 8. Assume that $P, Q \in t S N N I_{C}^{\phi}$ and that $P, Q$ are stable w.r.t. $\phi$. Then $(P \| Q) \in t S N N I_{C}^{\phi}$ and $P \| Q$ is stable w.r.t. $\phi$.

We have another compositionality principle for the $t G N D C_{\leq_{\text {ttrace }}}^{\alpha}$ schema, again under the assumption that the involved processes are stable.

Proposition 9. Given the set of initial knowledge $\phi$ and the set of public channels $C$, assume $P_{i} \in t G N D C_{\leq_{\text {ttrace }}}^{\alpha_{i}\left(P_{i}\right)}$, with $i=1,2$, and $P_{1}, P_{2}$ are stable w.r.t. $\phi$. It follows that $\left(P_{1} \| P_{2}\right) \in t G N D C_{\leq_{\text {ttrace }}\left(P_{1}\right) \| \alpha_{2}\left(P_{2}\right)}^{\alpha_{1}}$ and $P_{1} \| P_{2}$ is stable w.r.t. $\phi$.

One may wonder if the stability condition is too restrictive. As a matter of fact (see [11]), the above compositional proof principles can be successfully applied for checking integrity in stream signature protocols, as the ones in $[10,3]$.

\section{Conclusions}

We have shown how to extend the $G N D C$ schema to a real time setting while preserving the properties of the untimed schema. In particular, we have shown the existence of a "most powerful" timed enemy, at least for the timed trace semantics. We have also shown how to express uniformly in this general schema some timed security properties, such as timed Non Deducibility on Compositions, (one definition of) timed authentication, timed secrecy and also timed integrity. We have also introduced a compositional proof principle that allows us to compose safely two real-time security protocols, preserving the security properties they enjoy.

Related literature on real-time security include the prominent papers [16,2]. The former paper presents tock-CSP - a real-time language similar to tSPA - that is used 
to specify real-time crytpographic protocols. The main differences consists of a different treatment of timed operators, in the absence of a mechanism for handling cryptoprimitives, in the lack of a uniform schema, and in the absence of compositionality results. The latter paper [2] is mainly concerned with the model checking of the interesting case study of TESLA, a protocol for stream broadcasting over the internet. The main focus is on showing that it is possible to give a finite model for the unbounded supply of fresh cryptographic keys used during the protocol. The so-called security condition of the protocol is similar to timed agreement.

Compositional proof techniques for reasoning about cryptographic protocols in an untimed setting may be found in [1,13]. In [1], a compositional proof system for an environment-sensitive bisimulation has been developed. One main difference from ours, is that we consider a weak notion of observation where the internal actions are not visible. This permits us to have more abstract specifications. (As a matter of fact, the authors of [1] leave as future work the treatment of such a form of weak equivalence.) In [13], the authors develop the concept of disjoint encryption and, under this hypothesis, are able to perform compositional reasoning both for secrecy and authentication properties. While on the one hand, their approach seems to deal better with authentication properties than ours, on the other one it seems that there are situations where stability holds while disjoint encryption does not. (A deeper comparison deserves more time and space and is left as future work.)

Future work will be also devoted to study other security properties in a timed setting, such as non repudiation, for which apparently there is the need for using semantics more discriminating than timed trace semantics.

Acknowledgments. We would like to thank the anonymous referees for their helpful comments for the preparation of the final version of this paper.

\section{References}

1. M. Boreale and D. Gorla. on Compositional Reasoning in the Spi-calculus In proc. of FOSSACS'02, LNCS 2303, 2002.

2. P. Broadfoot and G. Lowe. Analysing a Stream Authentication Protocol using Model Checking In Procs. ESORICS'02, LNCS, Springer, 2002.

3. N. De Francesco and M. Petrocchi. Authenticity in a Reliable Protocol for Mobile Computing. Accepted at 18th ACM Symposium on Applied Computing (SAC03). U.S., March 2003.

4. R. Focardi and R. Gorrieri. A classification of security properties. Journal of Computer Security, 3(1):5-33, 1995.

5. R. Focardi, R. Gorrieri and F. Martinelli. A comparison of three authentication properties. To appear on Theoretical Computer Science.

6. R. Focardi, R. Gorrieri and F. Martinelli. Secrecy in Security Protocols as Non Interference. Electronic Notes in Theoretical Computer Science, 32. (2000)

7. R. Focardi, R. Gorrieri and F. Martinelli. Non-interference for the analysis of cryptographic protocols. In Procs. Int.l Colloquium on Automata, Languages and Programming (ICALP'00), LNCS 1853, 354-372, Springer, 2000.

8. R. Focardi, R. Gorrieri and F. Martinelli. Real-time Information Flow Analysis Journal of Selected Areas of Communications, IEEE Press, 2002, to appear. 
9. R. Focardi and F. Martinelli. A uniform approach for the definition of security properties. In Proceedings of World Congress on Formal Methods (FM'99), pages 794-813. Springer, LNCS 1708, 1999.

10. R. Gennaro and P. Rohatgi. How to Sign Digital Streams. Information and Computation 165(1), pp.100-116 (2001).

11. R. Gorrieri, F. Martinelli, M. Petrocchi and A. Vaccarelli. Using compositional reasoning and Non-interference for verifying integrity in digital stream protocols. Technical Report IIT, 2003. Submitted for publication.

12. J.F. Groote. Transition system specifications with negative premises. Theoretical Computer Science, 118:263-299, 1993.

13. J. Guttman and F.J. Thayer. Protocol Independence through Disjoint Encryption. In Proc. CSFW'00. IEEE Press, 2000.

14. G. Lowe. A Hiearchy of Autentication Specifications. In Proc. CSFW'97, pp. 31-43, IEEE Press.

15. R. Milner. Communication and Concurrency. Prentice Hall, 1989.

16. S. Schneider. Analysing Time-Dependent Security Properties in CSP using PVS. In Procs. ESORICS, LNCS 1895, 2000.

17. I. Ulidowski and S. Yuen. Extending process languages with time. In Proceedings of AMAST, LNCS 1349, pages 524-538, 1997. 